\title{
A Target-Detecting Visual Neuron in the Dragonfly Locks on to Selectively Attended Targets
}

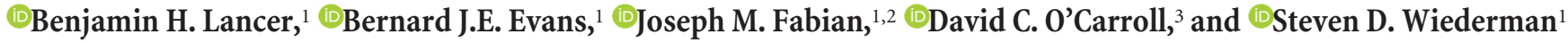 \\ ${ }^{1}$ Adelaide Medical School, The University of Adelaide, Adelaide, Australia 5005, ${ }^{2}$ Department of Bioengineering, Imperial College London, London SW7 \\ 2AZ, United Kingdom, and ${ }^{3}$ Department of Biology, Lund University, Lund, Sweden 22362
}

The visual world projects a complex and rapidly changing image onto the retina of many animal species. This presents computational challenges for those animals reliant on visual processing to provide an accurate representation of the world. One such challenge is parsing a visual scene for the most salient targets, such as the selection of prey amid a swarm. The ability to selectively prioritize processing of some stimuli over others is known as 'selective attention'. We recently identified a dragonfly visual neuron called 'Centrifugal Small Target Motion Detector 1' (CSTMD1) that exhibits selective attention when presented with multiple, equally salient targets. Here we conducted in vivo, electrophysiological recordings from CSTMD1 in wild-caught male dragonflies (Hemicordulia tau), while presenting visual stimuli on an LCD monitor. To identify the target selected in any given trial, we uniquely modulated the intensity of the moving targets (frequency tagging). We found that the frequency information of the selected target is preserved in the neuronal response, while the distracter is completely ignored. We also show that the competitive system that underlies selection in this neuron can be biased by the presentation of a preceding target on the same trajectory, even when it is of lower contrast than an abrupt, novel distracter. With this improved method for identifying and biasing target selection in CSTMD1, the dragonfly provides an ideal animal model system to probe the neuronal mechanisms underlying selective attention.

Key words: predictive gain modulation; target detection; insect vision; priming; selective attention; winner-takes-all

\section{Significance Statement}

We present the first application of frequency tagging to intracellular neuronal recordings, demonstrating that the frequency component of a stimulus is encoded in the spiking response of an individual neuron. Using this technique as an identifier, we demonstrate that CSTMD1 'locks on' to a selected target and encodes the absolute strength of this target, even in the presence of abruptly appearing, high-contrast distracters. The underlying mechanism also permits the selection mechanism to switch between targets mid-trial, even among equivalent targets. Together, these results demonstrate greater complexity in this selective attention system than would be expected in a winner-takes-all network. These results are in contrast to typical findings in the primate and avian brain, but display intriguing resemblance to observations in human psychophysics.

\section{Introduction}

The visual world contains a wealth of information about the environment and surroundings, yet even the most sophisticated visual systems lack the capacity to encode all the information in a

\footnotetext{
Received June 18, 2019; revised Sept. 4, 2019; accepted Sept. 6, 2019.

Author contributions: B.H.L., B.J.E.E., J.F., D.O., and S.D.W. designed research; B.H.L., B.J.E.E., and J.F. performed research; B.H.L. and B.J.E.E. analyzed data; B.H.L. wrote the first draft of the paper; B.H.L., B.J.E.E., J.F., D.O., and S.D.W. edited the paper; B.H.L. wrote the paper.

This work was supported by the Australian Research Council's Future Fellowship Scheme (FF180100466), the Australian Government Research Training Program (RTP), the Swedish Research Council (VR 2014-4904 and VR 2018-03452), and the Swedish Foundation for International Cooperation in Research and Higher Education (STINT). We thank the manager of the Adelaide Botanic Gardens for allowing insect collection.

The authors declare no competing financial interests.

Correspondence should be addressed to Benjamin Lancer at benjamin.lancer@adelaide.edu.au.

https://doi.org/10.1523/JNEUROSCI.1431-19.2019

Copyright $\odot 2019$ the authors
}

scene over time. Instead, animals must parse a scene for behaviorally relevant information and discard the remaining clutter. One solution to this problem is selective attention, the ability to selectively respond to one stimulus among multiple alternatives. Selective attention is observed across taxa, from humans and other primates (Treue, 2001) to insects (de Bivort and van Swinderen, 2016; Nityananda, 2016). Selective attention is particularly important in visual predatory animals, such as the dragonfly, which hunt among swarms containing potentially hundreds of prey and conspecifics (Edman and Haeger, 1974; Baird and May, 1997). Many predators hunting in these conditions are susceptible to the 'confusion effect', a reduced success rate due to difficulty tracking a single target amid the swarm (Landeau and Terborgh, 1986, Jeschke and Tollrian, 2007). Some dragonfly species, however, show particularly good performance hunting among swarms (Jeschke and Tollrian, 2007; Combes et al., 2012). 
Successful prey capture relies on the ability to filter irrelevant information, such as background clutter and conspecifics, while selecting and tracking prey among equally valuable alternatives. The confusion effect is diminished where predators are able to identify individual prey (Landeau and Terborgh, 1986). To achieve this, the underlying neuronal system should be able to 'lock on' to an individual target, while also being capable of switching targets when this would increase the chance of success.

We have previously identified an individual visual neuron in the dragonfly optic lobe that exhibits a 'winner-takes-all' selective attention (Wiederman and O'Carroll, 2013). Named "Centrifugal Small-Target Motion Detector 1" (CSTMD1), this binocular, efferent neuron resides in the optic lobes and midbrain (Geurten et al., 2007) and is thought to represent the output integrator of a network comprised of many lower-order, small-target motion detector neurons (STMDs). CSTMD1 is tuned for the movement of small $\left(1^{\circ}-3^{\circ}\right)$ dark targets against a bright background (O'Carroll, 1993; Geurten et al., 2007), matching the demands of an ethologically relevant target-detection system (Labhart and Nilsson, 1995; Olberg et al., 2005, 2007). CSTMD1's receptive field spans the whole visual field, but exhibits a sharp distinction between excitatory (contralateral relative to recording site) and inhibitory (ipsilateral) visual hemispheres (Geurten et al., 2007). When presented with two targets in the excitatory receptive field, CSTMD1 encodes the absolute strength of the selected target without interference from distracters (Wiederman and O'Carroll, 2013). In contrast, typical findings in primates (e.g., Recanzone et al., 1997; Treue and Maunsell, 1999), owls (Asadollahi et al., 2010) and other insects (Tang and Juusola, 2010; van Swinderen, 2012) show a response that is modulated by the presence of distracters. Encoding an absolute representation of a selected target (i.e., ignoring the distracter) has been observed in the auditory system of crickets (Pollack, 1988) and in primate MT neurons (Harrison et al., 2013). An analog exists in humans termed 'inattentional blindness', whereby an object in the visual field is ignored when attention is focused elsewhere (Simons and Chabris, 1999).

Previously, we have shown that CSTMD1 exhibits properties important for a prey-tracking system. First, the observation that selection could sometimes switch between targets mid-way through a trial (Wiederman and O'Carroll, 2013) raised the possibility that an ongoing competitive mechanism drives selection, even after an initial target has been selected, and that this mechanism can direct switches at opportune moments. Second, CSTMD1 exhibits 'predictive gain modulation' whereby a local facilitatory 'spotlight' of increased gain spreads forward along the predicted trajectory of a target (even accounting for occlusions), with inhibition elsewhere in the receptive field (Dunbier et al., 2012; Wiederman et al., 2017). This facilitation may represent a mechanism for 'locking-on' to a selected target, for example, a chosen fruit fly in a swarm.

Here, we have developed a technique to frequency tag targets by exploiting the contrast-dependent neuronal response (O'Carroll and Wiederman, 2014), permitting us to determine which target has been selected at any moment. Frequency tagging has previously been used during higher-order brain measurements (e.g., EEG) and in extracellular recordings measuring local field potentials (LFPs) in insects (van Swinderen, 2012). However, it is not yet known whether frequency components within these frequency tagged signals originate at the level of single neurons, or are an emergent property of a neuronal population code. To our knowledge, here we present the first application of this identification technique at the intracellular level. We thus demonstrate that, due to the contrast-sensitivity of CSTMD1, the frequency component of the stimulus is preserved in the individual neuron's response.

Applying this technique to intracellular spike trains, we show that CSTMD1 is both able to switch selected targets mid-trial and lock on to selected targets, even in the presence of a higher contrast distracter. We therefore describe a neuronal system more complex than the traditionally modeled winner-takes-all framework. This provides important insight into how selective behaviors are implemented by underlying neuronal processing.

\section{Materials and Methods}

Experiment preparation. We recorded from a total of 26 male, wildcaught dragonflies (Hemicordulia tau). Dragonflies were stored at $7^{\circ} \mathrm{C}$ for up to $7 \mathrm{~d}$ before experimentation. Dragonflies were warmed and then immobilized to an articulating magnetic stand with a 50/50 wax-rosin mixture. The head was tilted forwards to allow access to the back of the headcase, and a small hole was dissected in the rear of the head capsule adjacent to the esophagus to allow visual and physical access to the lobula complex and lateral midbrain.

We pulled aluminosilicate electrodes (Harvard Apparatus) using a Sutter Instruments P-97 electrode puller, which were filled with a $2 \mathrm{M} \mathrm{KCl}$ solution. Electrodes were then inserted into the lobula complex using a piezo-electric stepper with a typical resistance of 40-140 M $\Omega$. Intracellular responses were digitized at $5 \mathrm{kHz}$ for offline analysis with MATLAB.

There are two mirror-symmetric CSTMD1 neurons in each dragonfly brain, with one cell body residing in each hemisphere. We record from the left optic lobe where a tract containing a large-diameter section of the contralateral CSTMD1's axon is known to reside. We can therefore record from a maximum of one CSTMD1 per dragonfly.

Visual stimuli. We presented stimuli on high-definition LCD computer monitors $(120-165 \mathrm{~Hz})$ using a custom-built presentation and data acquisition suite based on MATLAB (RRID:SCR_001622) and Psychtoolbox (RRID:SCR_002881. Available at: http://psychtoolbox.org/). The animal was placed $20 \mathrm{~cm}$ away from the monitor and centered on the visual midline, thus minimizing off-axis artifacts. Stimuli consisted of a single or pair $\left(\sim 20^{\circ}\right.$ separation $)$ of $1.5^{\circ}$ by $1.5^{\circ}$ squares of modulated contrast ascending the receptive field at a speed of $40 \%$.

We applied to our intracellular recordings a frequency tagging paradigm inspired by human electroencephalography research (Norcia et al., 2015) and local field potential research in insects (van Swinderen, 2012). We presented two competing, flickering targets each with varying contrast at two different frequencies. As neuronal responses are themselves modulated by the contrast, spikes become entrained to the high contrast phase of the flicker so that modulation of the observed response permits identification of the selected target. To test that the technique was not dependent on the choice of the tagging frequency (i.e., used only for identification and not saliency), we presented nonharmonic frequencypairs of either $8 \mathrm{~Hz}\left(F_{1}\right)$ and $12\left(F_{2}\right) \mathrm{Hz}$, or $11 \mathrm{~Hz}\left(F_{1}\right)$ and $15\left(F_{2}\right) \mathrm{Hz}$. These frequencies were not multiples of one other but were divisible by the monitor refresh rate, thus ensuring the full range of intensities were presented within each period. We tested with both sinusoidal and square wave flicker. These results were subsequently pooled because there was no discernible difference in their power to identify selection.

Frequency tagged targets flickered between a minimum Weber contrast of 0.06 and maximum of 1 (mean contrast of 0.51 and a white background of $337 \mathrm{Cd} / \mathrm{m}^{2}$ ). In single target trials, one target contrast varied at either $\mathrm{F}_{1}, \mathrm{~F}_{2}$, or $0 \mathrm{~Hz}$ (i.e., a nonflickering control at maximum contrast) and was presented moving vertically up the display at one of two spatial locations, $\mathrm{T}_{1}$ or $\mathrm{T}_{2}$ (locations separated $20^{\circ}$ horizontally within CSTMD1's excitatory, receptive field). In paired-target trials, targets of different flicker frequencies were simultaneously presented at both $\mathrm{T}_{1}$ and $\mathrm{T}_{2}$ locations. The choice whether the spatial location $\mathrm{T}_{1}$ or $\mathrm{T}_{2}$ was either $\mathrm{F}_{1}$ or $\mathrm{F}_{2}$ (e.g., $8 \mathrm{~Hz}$ or $12 \mathrm{~Hz}$ ), was pseudo-randomized to control for any preferred frequency response.

Experimental design and statistical analysis. For the trial by trial selection processes, any given trial must be considered an independent event as averaging (as in technical replicates) would mask the observation. However, to ensure statistical robustness of the result we 

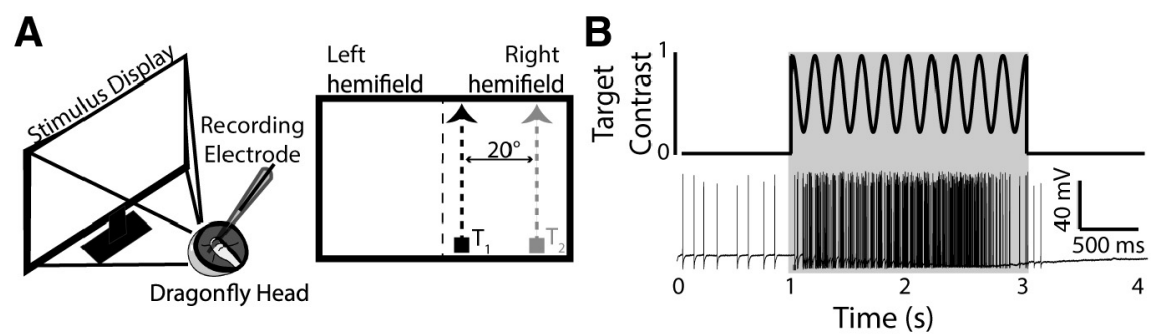

C

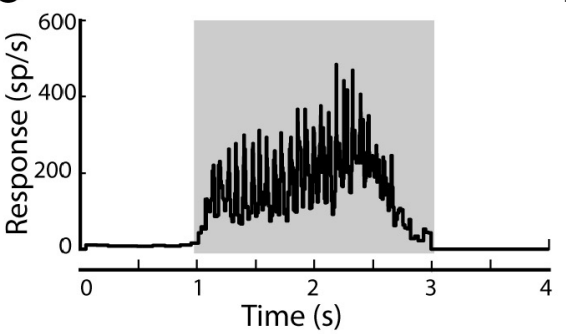

E
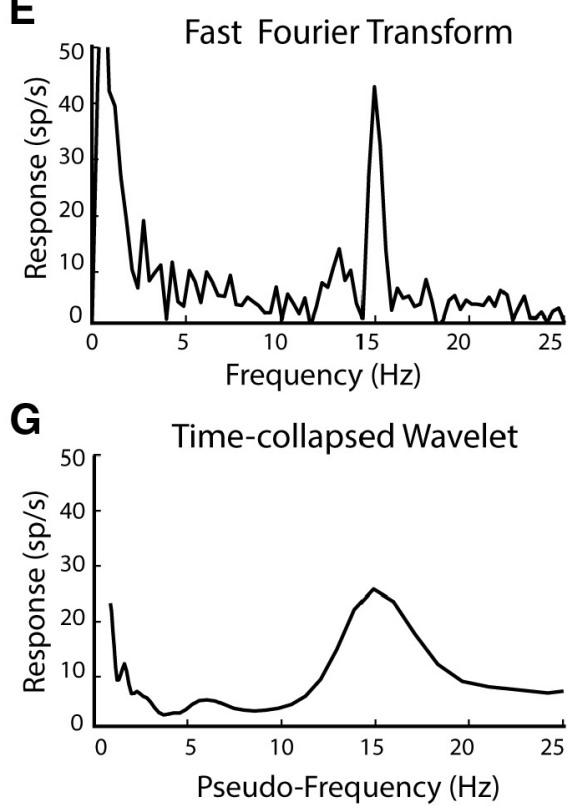

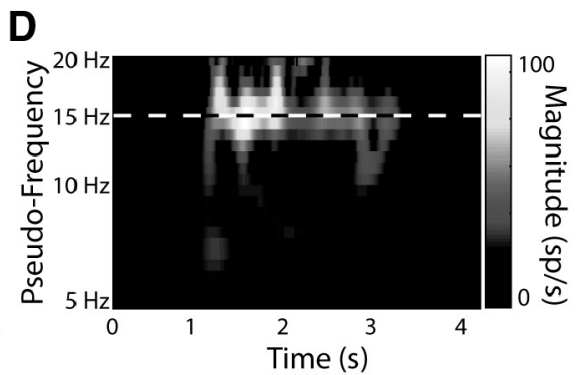

$\mathbf{F}$

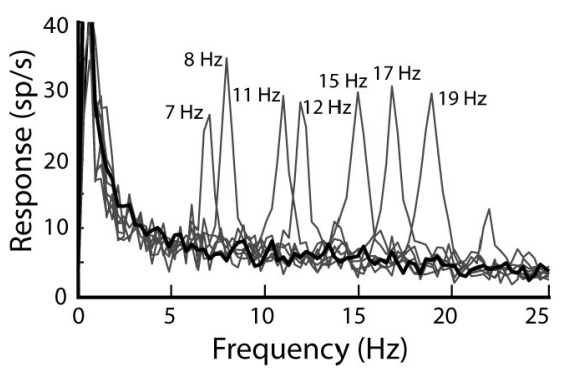

H

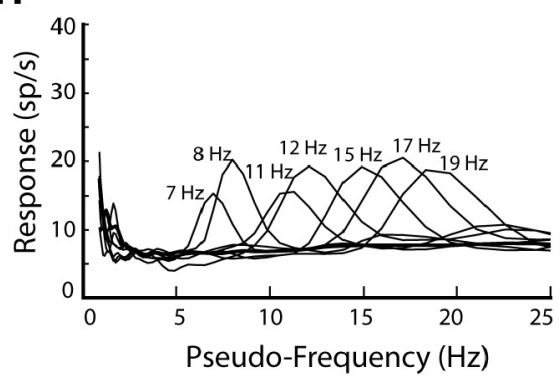

Figure 1. The frequency of the tagged target is preserved in the intracellular responses of CSTMD1. A, Left, Intracellular in vivo electrophysiology involves inserting an electrode into the intact brain to record single-neuron responses to stimuli presented on a computer screen. Right, Stimulus pictogram, a single small target (black) ascends CSTMD1's excitatory receptive field. Relative positions of $\mathrm{T}_{1}$ (black) and $\mathrm{T}_{2}$ (gray) indicated. $\boldsymbol{B}$, Top, Frequency tagging involves modulating the contrast of the stimulus over time at a specific frequency ( $5 \mathrm{~Hz}$ in this illustrative pictogram). Bottom, An example spike train in response to a stimulus modulated at $15 \mathrm{~Hz}$, presented at $1 \mathrm{~s}$ for a duration of $2 \mathrm{~s}$. C, The inverse interspike interval (ISI) is calculated to determine the spike rate over time. This calculation provides a continuous signal that is amenable to frequency-domain analysis. $\boldsymbol{D}$, A continuous wavelet transform of the signal in ( showing magnitude across time and pseudo-frequency (Logarithmic) reveals response magnitude at a range of pseudo-frequencies over time. In this example, power is centered around $15 \mathrm{~Hz}$ for the duration of the trial. $\boldsymbol{E}, \mathrm{A}$ Fast Fourier Transform of the signal in ( reveals a distinctive peak at $15 \mathrm{~Hz}$, corresponding to the frequency tagged stimulus. $F$, Averaged FFT of responses to trials of varying frequency ( $n=119$ trials across 4 dragonflies) $G$ ) The output of the wavelet analysis in $D$, collapsed across time to be visually comparable to the FFT in $\boldsymbol{E}$. $\boldsymbol{H}$, Averaged time-collapsed continuous wavelet transform for the same data presented in $\boldsymbol{F}$, which although less peaked, still reveals statistically distinctive responses at the relevant frequencies.

repeated experiments across several dragonflies. Here we use ' $n$ ' to denote the number of trials and additionally report the number of dragonflies. We visualize all trial data points and describe similarities or differences across animals.

We report exact $P$ except when $<0.001$. All tests are nonparametric, two-tailed and corrected for multiple comparisons (Bonferroni-Holm correction). Box and Whisker plots indicate median, interquartile and minimum/maximum range. Unless otherwise stated outliers are indicated with $\Delta$.
All data analysis was conducted in MATLAB 2017a (RRID:SCR_001622), including the Wavelet Toolbox. Continuous wavelet transforms (CWTs) used an analytic Morlet wavelet with gamma $=3$.

\section{Results}

Neuronal responses can be frequency tagged

To test the validity of the frequency tagging technique, we presented a single flickering target moving vertically up the display within the dragonfly's field of view (Fig. 1A). The target ascended at $40 \%$ s within the excitatory region of CSTMD1's receptive field (Wiederman and O'Carroll, 2013; Wiederman et al., 2017). We use the term 'frequency tagging' to refer to the modulation of Weber contrast: (Intensity target $_{-}$ Intensity background $_{\text {(Intensity }}$ / background $_{\text {, }}$, over time at a set frequency (in Hertz). Since CSTMD1 is selective for dark targets (Wiederman et al., 2013), we flickered a black-to-gray target against a white background (Fig. 1B, top). An example of an individual data trace in response to a 15 $\mathrm{Hz}$ target shows the spike activity during the stimulus presentation (Fig. $1 B$, bottom). To extract any frequency tagged response modulation, we first determine spike locations and calculate the instantaneous spike rate (Inverse Inter-Spike Interval) over time (Fig. 1C). We applied two mathematical transforms to these data; a Continuous Wavelet Transform (Fig. $1 D$ ), and a Fast Fourier Transform (Fig. $1 E)$. The application of a Fast Fourier Transform (square root to provide amplitude) reveals a peak modulation of the neuronal rate code in the frequency domain at $15 \mathrm{~Hz}$, equivalent to the target contrast modulation (a response at $0 \mathrm{~Hz}$ is due to the nonzero mean over time). We repeated this process for a series of different frequencies (averaged across neurons) to determine appropriate frequencies for the experiments (Fig. $1 F$ ). These data show that from 7 to $19 \mathrm{~Hz}$ the frequency content of the stimulus is well preserved in the intracellular response of single neurons. However, we have previously shown that CSTMD1 can 'switch' selection midtrial (Wiederman and O'Carroll, 2013). In this circumstance, power from the FFT would be distributed between the two target frequencies, corresponding to the total time each target was selected. Therefore, Fourier analysis of the entire spike train cannot distinguish between: (1) trials where modulation was genuinely shared between $T_{1}$ and $T_{2}$ (indicative of a lack of competitive selection, such as neuronal summation) or (2) those where selection switched from $T_{1}$ to $T_{2}$ or $T_{2}$ to $T_{1}$ part-way through the trial. To account for possible switches, we instead applied CWTs that provides power across pseudofre- 
quencies over time (Fig. 1D). Averaging the wavelet analysis across time is comparable to a FFT, though reveals a broader peak in the frequency domain centered at $15 \mathrm{~Hz}$ (Fig. $1 G$ ). The broader shape observed in the CWT is inherent to the wavelet analysis and is the cost of providing information of how frequency components vary over time. Although in the frequency domain CWT responses are blurred compared with their FFT counterparts, there are statistically significant differences for any two frequencies separated by at least $2 \mathrm{~Hz}(p<0.001)$. Thus, we were able to analyze all further data using CWTs to derive the benefit of examining the frequency response evolution over time of the individual trials.

Frequency tagging reveals which target is selectively attended To test the ability of the frequency tagging technique to discriminate selected and unselected targets, we first attempted to reproduce our earlier demonstration of selective attention in CSTMD1 (Wiederman and O'Carroll, 2013), where the response to two competing targets presented simultaneously closely resembles the unique response for the individual targets presented alone. To this end we presented either single targets (pseudo-randomly at either $f_{1}$ or $f_{2}$ ) at either spatial location $T_{1}$ or $T_{2}$ (both within CSTMD1's excitatory receptive field). Randomly interleaved with the single target trials (Fig. $2 A$ ), we also presented pairedtargets (i.e., simultaneously at both target locations $\mathrm{T}_{1}$ and $\mathrm{T}_{2}$ ) which were frequency-modulated at the two different frequencies (pseudo-randomly between $\mathrm{T}_{1}=\mathrm{f}_{1}, \mathrm{~T}_{2}=\mathrm{f}_{2}$ and $\mathrm{T}_{1}=\mathrm{f}_{2}$ and $\mathrm{T}_{2}=$ $\left.f_{1}\right)$. As our interest is in the chosen target $\left(T_{1}\right.$ or $\left.T_{2}\right)$, rather than the frequency of the 'identifier', we pooled data across the frequency-pairs.

In single-target trials (Fig. $2 B$, location $\mathrm{T}_{1}$ orange dots; location $\mathrm{T}_{2}$ blue dots), we usually observed strong modulation at the frequency of the presented target and weak modulation at the alternative frequency (i.e., a frequency that does not exist in the stimulus, therefore representing a form of experimental and analysis noise). However, some individual trials had insufficient modulation in the transform to enable accurate identification of the selected targets. This likely results from two factors: (1) neuronal habituation in the receptive field diminishing the strength of the modulation, or; (2) neuronal saturation from a highly responsive cell limiting the possible strength of the modulation. To analyze trials free of these effects, we used single-target responses to determine a threshold for data inclusion. For each location, $\mathrm{T}_{1}$ and $\mathrm{T}_{2}$, we calculated the average magnitude at the frequency not presented, which provides an estimate of the noise inherent in the frequency domain. This floor was defined as the mean power at the nonpresented frequency plus twice the standard deviation. This provided an objective level of the modulation noise at the other frequency. That is, the expected, nonzero modulation at $f_{2}$ when the neuron has selected a target modulated at $f_{1}$, and viceversa (Fig. $2 B$, dashed lines). Trials in the bottom-left corner of Figure $2 B$ thus fail the acceptable signal-to-noise threshold for both frequencies. Using this measure, we rejected 172 trials (27.6\% of the total) from any further analysis, revealing that our frequency tagging technique worked for $71.4 \%$ of the total trials presented. There was no significant difference in the number of identification failures between any of the three conditions $\left(\mathrm{X}^{2}\right.$ test, $p>1$, Bonferroni-Holm correction), therefore there was no effect of this data exclusion on the further testing of hypotheses with respect to the presence of selective attention. We applied these exclusion criteria to all further data analysis.
Responses above threshold, at either $\mathrm{f}_{1}$ or $\mathrm{f}_{2}$, indicated significant identification of either one, or both, of the targets. Qualitatively, we observe that the responses to paired-targets (Fig. 2B, crosses) were mostly either modulated at the frequency of the target at location $\mathrm{T}_{1}$ or $\mathrm{T}_{2}$ (but not both, i.e., only a few crosses within the 'Shared or Switch' region).

The absolute modulation above this noise threshold (i.e., the distance of the data points along the abscissa or ordinate in Fig. $2 B$ ) is related to the trial-by-trial sensitivity, rather than to the degree of the selective attention to one or either of the targets. To quantify our data, we therefore defined a selectivity index (Fig. $2 C$ ), which measured the degree of target selection, independent of the strength of response modulation (providing it is above the noise exclusion threshold as previously described). For each data point, we calculated the following:

$$
\text { Selectivity Index }=T_{1}-T_{2} / \sqrt{T_{1}^{2}+T_{2}^{2}}
$$

$\mathrm{T}_{1}$ and $\mathrm{T}_{2}$ values are averages of the pseudo-frequency amplitude (known as 'scale') over the trial duration (i.e., collapsed across time from the CWTs), for each of the corresponding target frequency tagging modulations. The selectivity index ranges between +1 and -1 and represents the selection of $\mathrm{T}_{1}(+1)$ and $\mathrm{T}_{2}$ $(-1)$, respectively. Here 'selectivity' is referred to in the original definition of 'selective attention' as selection of one from multiple competing stimuli, as would be expected in a winner-takes-all network. A value of 0 would occur if the response magnitude at $f_{1}$ and $f_{2}$ were equal (regardless of the absolute distance from the origin), indicating either shared (covarying) selection across the trial, or a switch in selection during the trial.

In Figure $2 C$, we observe significant differences in the selectivity index distribution between paired and both $\mathrm{T}_{1}$-alone and $\mathrm{T}_{2}$-alone conditions ( $p<0.001$, Bonferroni-Holm correction). In single-target conditions, the selectivity index is narrowly distributed $\left(\mathrm{T}_{1} \mu=0.68, \sigma=0.17 ; \mathrm{T}_{2} \mu=-0.58, \sigma=0.23\right.$ ), whereas in paired-target trials the selectivity index is nonnormally distributed ( $p>0.001$, one-tailed Kolmogrov-Smirnov test) with peaks at $\sim 0.65$ and $\sim-0.55$. The bimodal distribution of responses to paired-targets reveals the selection of either $\mathrm{T}_{1}$ or $\mathrm{T}_{2}$. For comparison to a potential "null" hypothesis (i.e., no selective attention), Figure $2 D$ shows results from a single lobula tangential cell in the dragonfly (Evans et al., 2019). This neuron generates robust responses using spatial summation to encode wide-field optic flow, analogous to lobula plate tangential cells in Diptera (Hausen, 1982). We presented the same experimental paradigm, though with larger targets $\left(1.5^{\circ} \times 10^{\circ}\right)$ to elicit a response. In contrast to the results observed in CSTMD1, the optic flow neuron had a selectivity index around 0 (modulation at both frequencies of the paired-targets) indicative of neuronal spatial summation.

\section{Target selection occasionally switches midway}

Not all of the paired-target trials were solely modulated by one of the target frequencies (Fig. $2 B$, shared zone). What could account for this apparent shared modulation? There are two possible explanations. First, that the neuron is excited by both stimuli at their respective frequencies and is not selecting a single target. That is, spatial summation similar to what is observed in the lobula tangential cell (Fig. 2D) and in primate V4 (Ghose and Maunsell, 2008). Second, a switch midway through the trial could result in significant modulation at both 
A
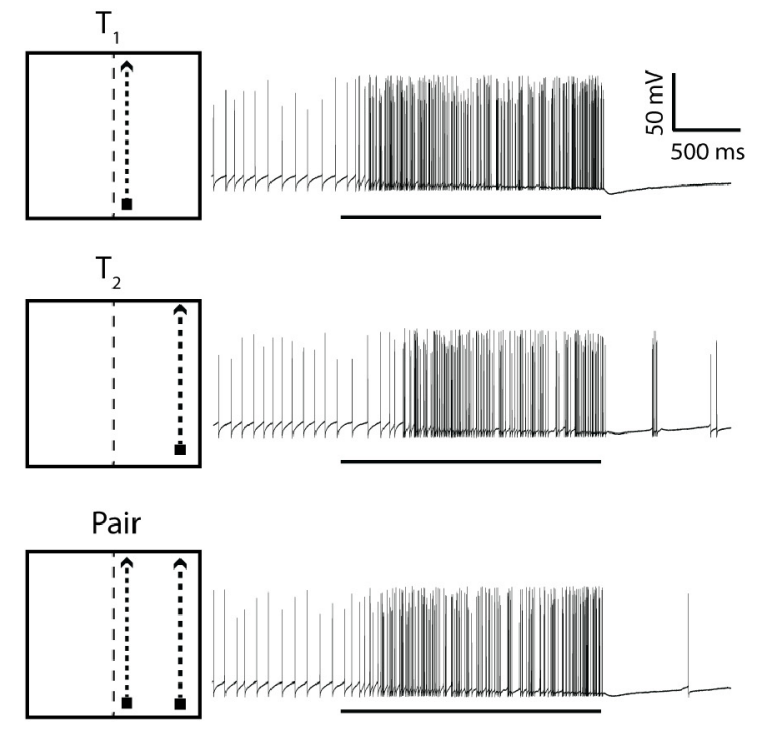

B

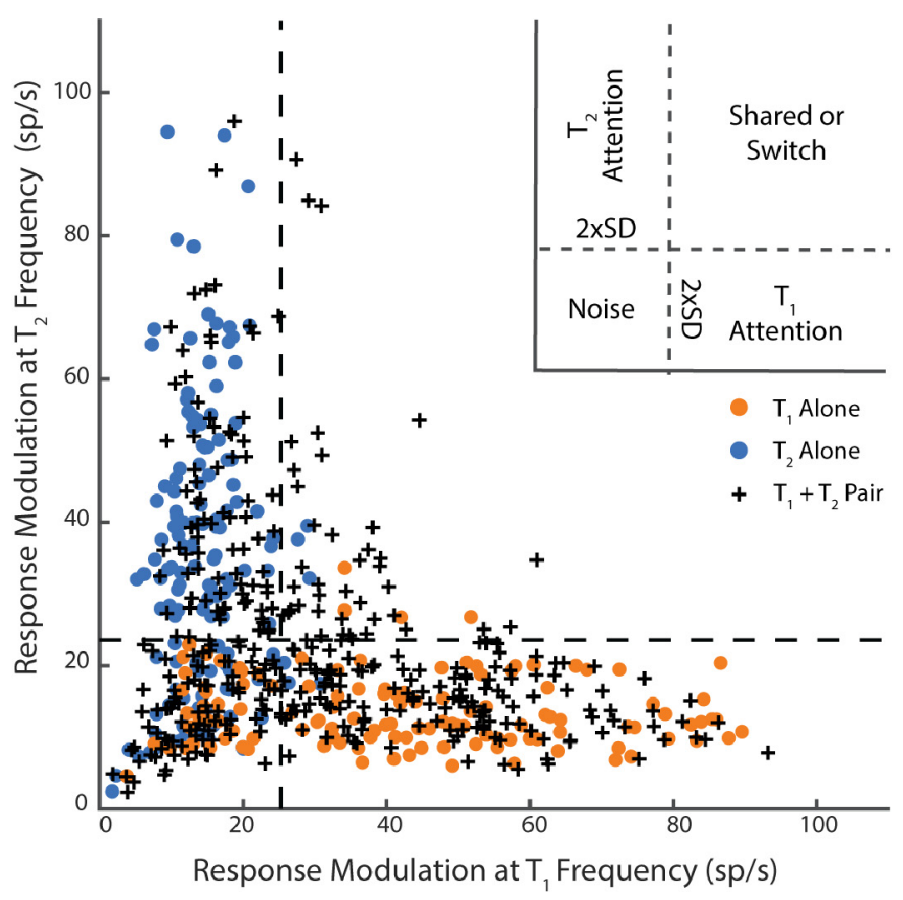

D Lobula Tangential Cell

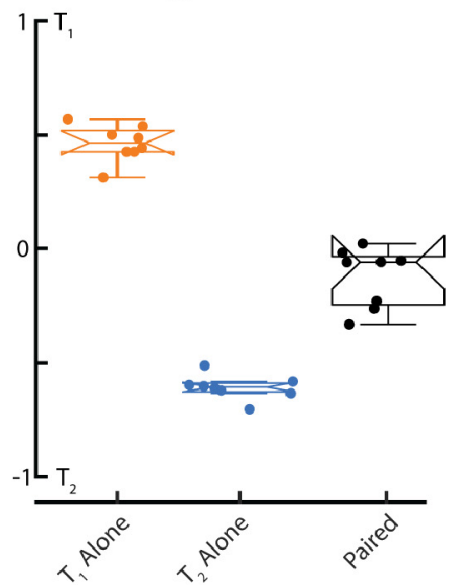

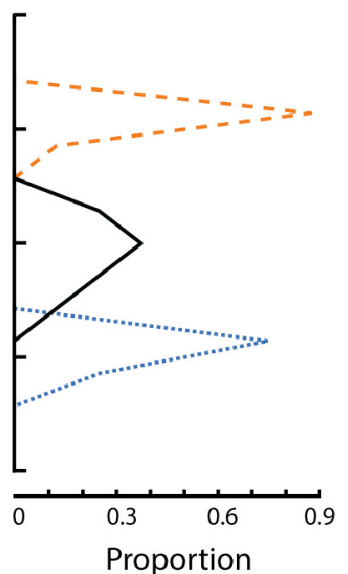

Figure 2. Frequency tagging identifies the selected target in a paired-target trial. $A$, Illustrative pictograms and corresponding single-trial electrophysiological responses for the three stimulus conditions. From top to bottom: $T_{1}$ alone, $T_{2}$ alone, paired targets. $\boldsymbol{B}$, The response modulation at the $T_{2}$ frequency plotted against response modulation at the $T_{1}$ frequency. Data are plotted in response to either a single target at the $\mathrm{T}_{1}$ location (orange dots) or at the $\mathrm{T}_{2}$ location (blue dots) when presented alone. Crosses represent CSTMD1 responses to the paired stimulus (total $n=447$ trials across 13 dragonflies). Dashed lines indicate the derived noise threshold. Responses to the paired-targets mostly elicit modulation at either one or other of the target flicker frequencies (not both together), indicative of selective attention. C, The selectivity index represents the degree to which the response is locked to one of the frequency tagged stimuli over the other. Values around zero indicate that both frequencies are equal components of the overall modulation. Frequency polygons illustrate the relative proportion of these points, with the bimodal distribution to the paired stimulus clearly revealing the selection of one target or the other. $\boldsymbol{D}$, In comparison, results from a lobula tangential cell (an optic flow sensitive neuron) in the dragonfly show no selective attention ( $n=8$ trials in 1 dragonfly), with a unimodal distribution around zero to the paired-targets, indicative of the expected shared modulation to both target frequencies (neuronal summation rather than selection).

frequencies, as both targets are selected during the trial, though at discrete times.

To differentiate between these potential explanations, we first simulated a switch in response from $\mathrm{f}_{1}$ to $\mathrm{f}_{2}$ by presenting a single-target that changed frequency in the middle of the trial (Fig. $3 A$ ). An example of the intracellular response to such a pseudo-switch stimulus is presented in Figure $3 B$. We then took a 'slice' from the Continuous Wavelet Transform at each frequency of interest $( \pm 1 \mathrm{~Hz})$ and subtracted these from one another (Fig. 3C, dashed lines), thus producing a difference in magnitude between the two pseudo-frequencies over time (Fig. 3D). This difference in magnitude provides a read-out through time of how much the modulation was determined by each target's frequency. A flat line near zero would indicate shared modulation distributed between the two frequencies.

We applied this 'difference slice' analysis to determine whether the paired-target responses with modulation at both frequencies (Fig. $2 B$, shared or switch region) were due to spatial summation or switching. Figure $4 A$ shows individual examples from six such trials, all of which exhibit discrete peaks and 
A

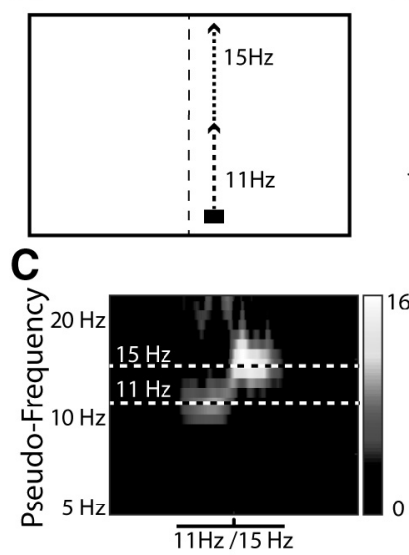

B
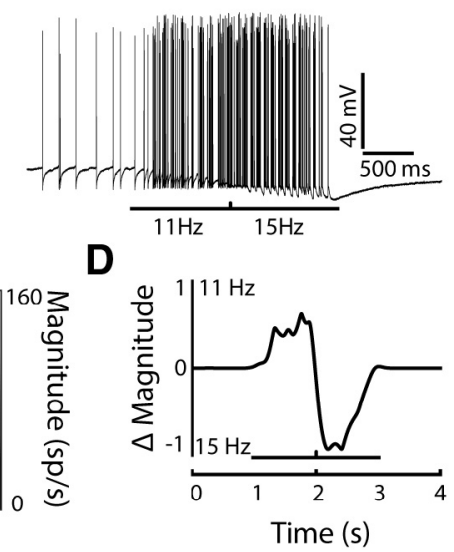

Figure 3. A simulation of an "attentional switch" is encapsulated by the difference between the pseudo-frequency (scale) magnitudes over time. $\boldsymbol{A}$, llustrated pictogram of a single target that changed frequency modulation halfway through the trial, simulating an attentional switch from one target to another. $\boldsymbol{B}$, A single-trial example of CSTMD1's response to this switching stimulus. C, The CWT of the inverse ISI of the trial in $\boldsymbol{B}$ reveals the switch that occurs halfway through the trial. The black-and-white dashed lines indicate the $11 \mathrm{~Hz}$ and $15 \mathrm{~Hz}$ frequency slices. D, A "difference slice" (delta magnitude) is calculated by taking the difference between the wavelet slices at 11 and $15 \mathrm{~Hz}$ across time.

troughs across time. The traces indicate that these CSTMD1 responses are switching between targets, rather than being modulated by both target frequency tags simultaneously.

To compare aggregate data, we calculated a 'Switch Index' for each trial (Fig. 4B). This index was calculated by determining the proportion of time the system selected either $T_{1}$ or $T_{2}$. To ensure that these selections were robust, we only considered a selection valid when either target was $>5$ spikes/s stronger than its counterpart. The time each target was selected was multiplied, thus if one of the targets was not selected, the Switch Index was zero. The Switch Index (normalized) is maximized when both targets are selected (not shared) for $50 \%$ of the trial. The Switch Index is low in single-target trials (orange or blue dots), since the time when the other target is selected is near zero. In paired-target trials the switch index is distributed between high and low values, representing either absolute selection of one target, or switches midway. In trials with a selectivity index around 0 (shared modulation), the Switch Index is uniformly high, indicating that the shared modulation results from switches in the selected target over the time course, rather than summation of responses to both targets. In comparison, paired-target trials in the control dragonfly lobula tangential cell, show both a low selectivity index and low Switch Index, indicating genuine modulation at both frequencies (simultaneously) over time due to the spatial summation used in optic flow computations (Fig. $4 B$, stars).

\section{Selection can be biased with priming}

We then tested the ability of a priming stimulus to bias the selection of a spatially associated target in a paired-target condition. In this experiment, a lone untagged primer was first presented for one second moving toward the trajectory of either spatial location $\mathrm{T}_{1}$ or $\mathrm{T}_{2}$ (Fig. $5 A$ ). Note that here the frequency-tagged $\mathrm{T}_{1}$ and $\mathrm{T}_{2}$ pathways commence midway up the stimulus display, immediately after the single "primer" target has moved along its trajectory. From our previous work, we expect CSTMD1 to predictively facilitate responses in front of the target's prior path (Nordström et al., 2011; Dunbier et al., 2012; Wiederman et al., 2017). We introduced a frequency tagged distracter midway through the receptive field (horizontally offset by $20^{\circ}$ ) paired with a frequency tagged target that continued along the primer's previous trajectory (Fig. 5A). We calculated the selectivity index across the period $(1 \mathrm{~s})$ where both targets are presented together and reveal a significant $(p<0.001)$ biasing of selection toward the target that continues along the primed trajectory (Fig. $5 B$ ). This selection may be due to "predictive gain modulation," whereby a local spotlight of enhanced gain is generated ahead of a moving target, with suppression in the surround (Wiederman et al., 2017). In our experiment, the continuing target is within the spotlight created by the preceding target, but the distracter appears within the suppressed surround.

In the human psychophysics literature, attentional capture is an effect whereby the presentation of an abrupt-onset stimulus (Yantis and Jonides, 1984) or a novel object (Franconeri et al., 2005) involuntarily captures attention (Remington et al., 1992), even when task-irrelevant. To test for a capture of CSTMD1's selection, we analyzed the previous biased paired-target responses (Fig. 5B) separated into three $400 \mathrm{~ms}$ periods (early, middle and late). We included 100 ms overlap between these periods because this duration was required for meaningful CWT analysis. If CSTMD1 responses displayed attentional capture, we hypothesize that the early period would be dominated by responses to the distracter stimulus, returning to the original path at later periods of time (as the distracter is assessed and ignored). Our results revealed the opposite effect (Fig. 5C), with the early window exhibiting the strongest biasing effect, which can dissipate over time (via switches). This reveals that selection is not captured by abrupt-onset novel stimuli presented within CSTMD1's receptive field. Rather responses are 'locked' to the preceding target's predicted continuing trajectory and generally ignore a novel distracter that falls outside of this predicted location. Here we observed asymmetry in results from the $\mathrm{T}_{1}$ compared with $\mathrm{T}_{2}$ priming, which reflects the broader (noisier) distribution of values in the $T_{1}$ primer condition when analyzed over the entire analysis duration (Fig. $5 B$ ). When primed to $\mathrm{T}_{1}$ (the target closer to the dragonflies' midline), the early window (Fig. 5C) reflects this biasing to the continued path trajectory (though note the exceptions). However, in some cases over time (middle and late windows) selection can change toward the distracter location at $T_{2}$. This results in significant changes in the selectivity index between these periods $(p<0.001)$. Visual inspection of the CWT analysis reveals that these are switches that occur at discrete points in time in the individual trials. In the $\mathrm{T}_{2}$ priming condition (the target located in the more peripheral location), the selection has locked on to the preceding target and maintains this selection throughout the rest of the trial, with no significant difference between the early, middle and late periods. Again, there are single-trial exceptions, however, these are distributed in either $\mathrm{T}_{1}$ or $\mathrm{T}_{2}$ selection.

\section{Selection can lock on to lower contrast targets}

In a traditional winner-takes-all network (Feldman and Ballard, 1982), the introduction of a higher contrast distracter during the presentation of a lower contrast target would result in a switch to the one with higher salience. However, how would the dragonfly feed in a swarm if often distracted by a novel, transiently more salient target? To determine whether CSTMD1 locks-on to the lower-salience stimuli, we presented primers of varying contrasts followed by introduction of a frequency-tagged distracter. We designed the lower contrast target to retain its lower saliency throughout the course of the trial (i.e., no frequency tagging), even during the period of the paired distracter. Hence the pres- 
A

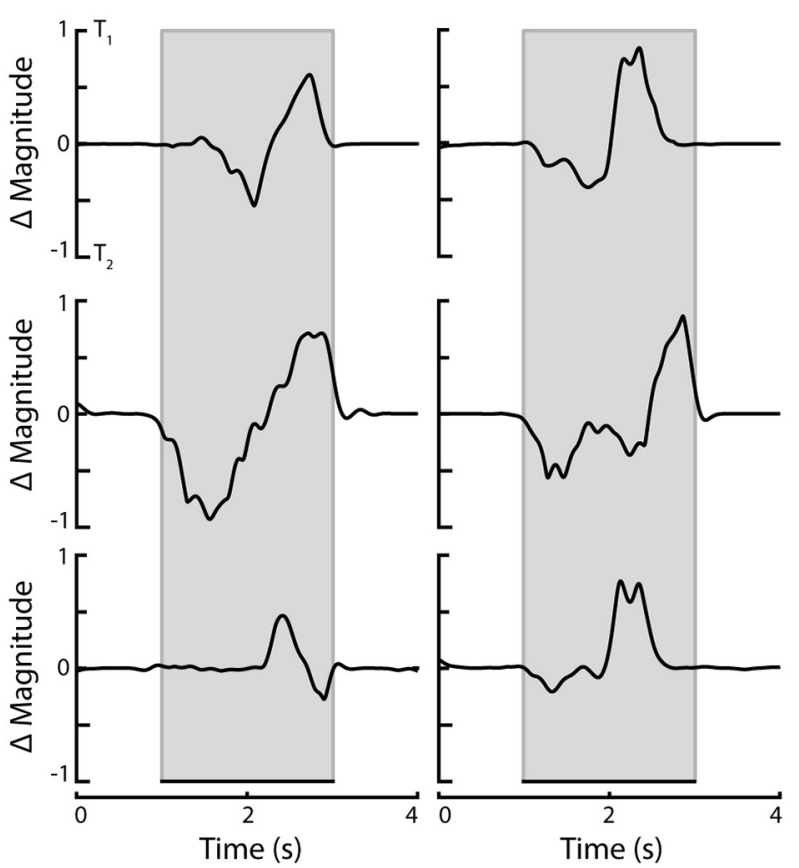

B

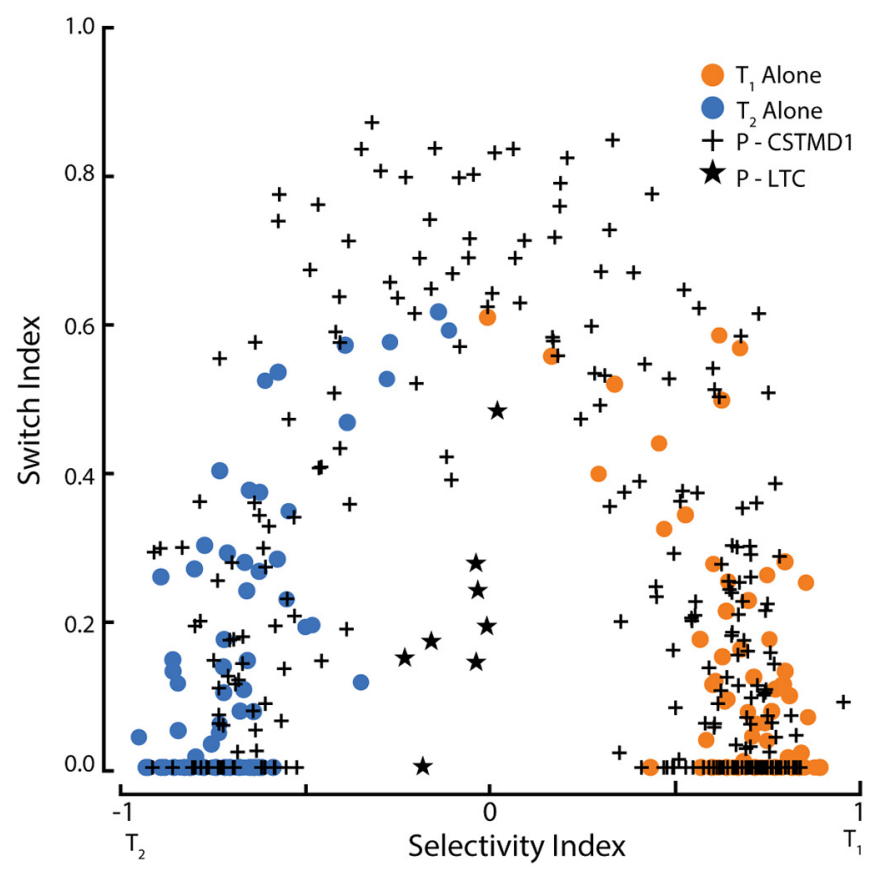

Figure 4. Shared modulation results from switches in selection over time. $\boldsymbol{A}$, Individual examples reveal high modulation for both targets, however only at different epochs of time. $\boldsymbol{B}$, The switch index and selectivity index for all single target (orange and blue points) and paired-target (crosses) trials from Figure $2 B$ (total $n=447$ trials across 13 dragonflies). When selectivity for paired-targets is low (middle abscissa, close to zero) then the Switch Index is high, indicating that responses switched between targets. In comparison, the lobula tangential cell (an optic flow neuron) (stars) has low selectivity and low switch, indicative of neuronal summation (modulation at both frequencies across points in time).

\section{A}

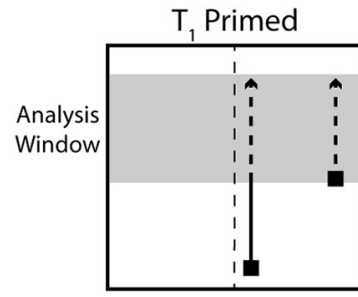

B

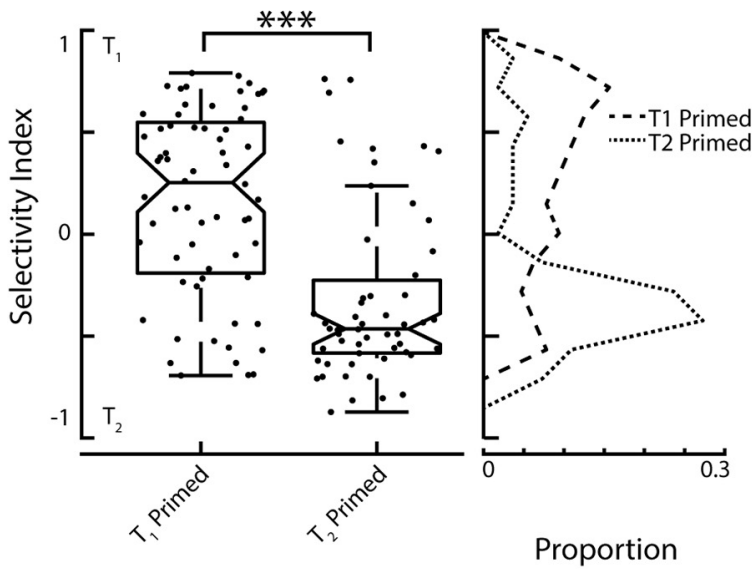

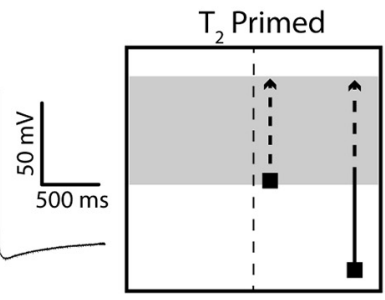

C
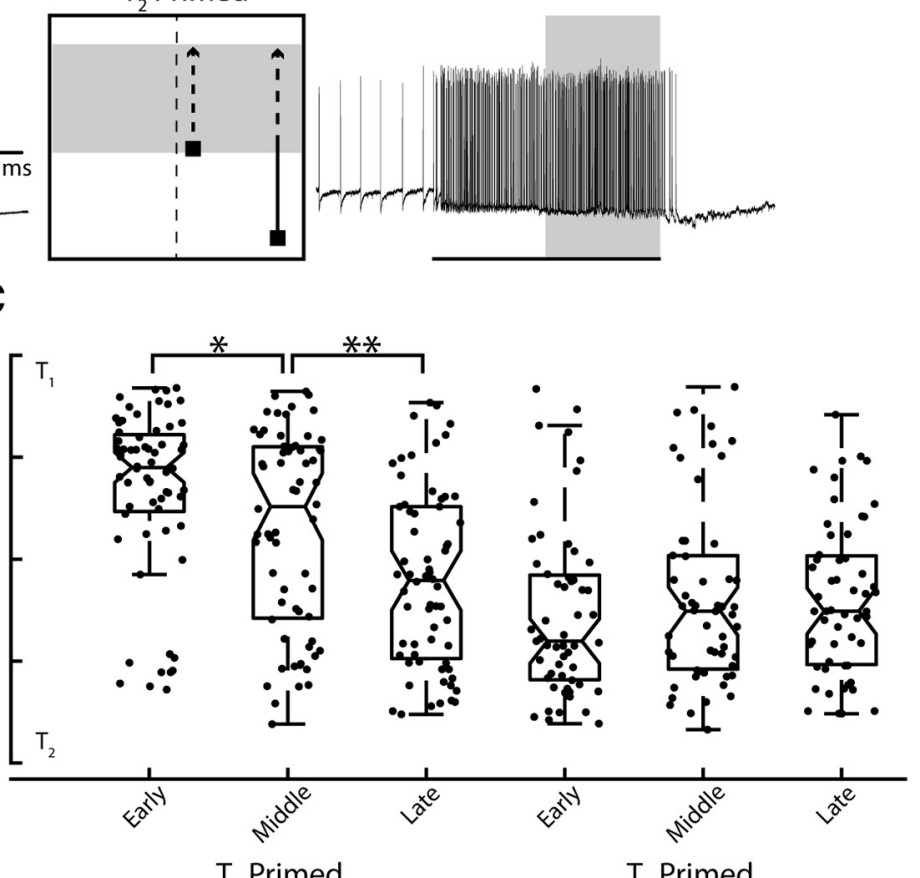

Figure 5. Priming with a preceding target biases selection toward the continuing trajectory. $A$, Pictograms illustrate the biasing stimulus toward either spatial location $T_{1}$ or $T_{2}$, next to individual examples of CSTMD1 responses. The short-path target (distracter) appears at $1 \mathrm{~s}$, when the preceding target reaches midway up the screen (the analysis window indicated with the gray shaded region). $\boldsymbol{B}$, There is a significant difference in the selectivity index between $\mathrm{T}_{1}$ and $\mathrm{T}_{2}$ primed trials ( $n=295$ across 7 dragonflies), though priming is not effective in all trials. Frequency polygons reveal the distributions of the selectivity index for $\mathrm{T}_{1}$ primed (dashed line) and $\mathrm{T}_{2}$ primed (dotted line). $C$, The selective attentional capture from priming split over early, middle and late time windows. Over time, $\mathrm{T}_{1}$ primed selection shifts to $\mathrm{T}_{2}$, while $\mathrm{T}_{2}$ selection is retained over the three periods. ${ }^{*} p \leq 0.05,{ }^{* *} p \leq 0.01,{ }^{* * *} p \leq 0.001$. 
A
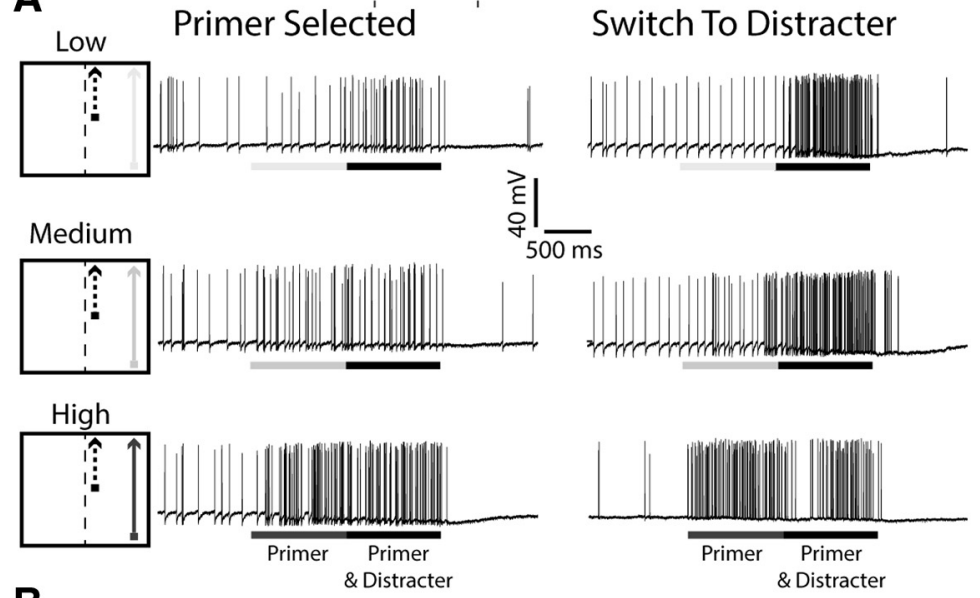

B
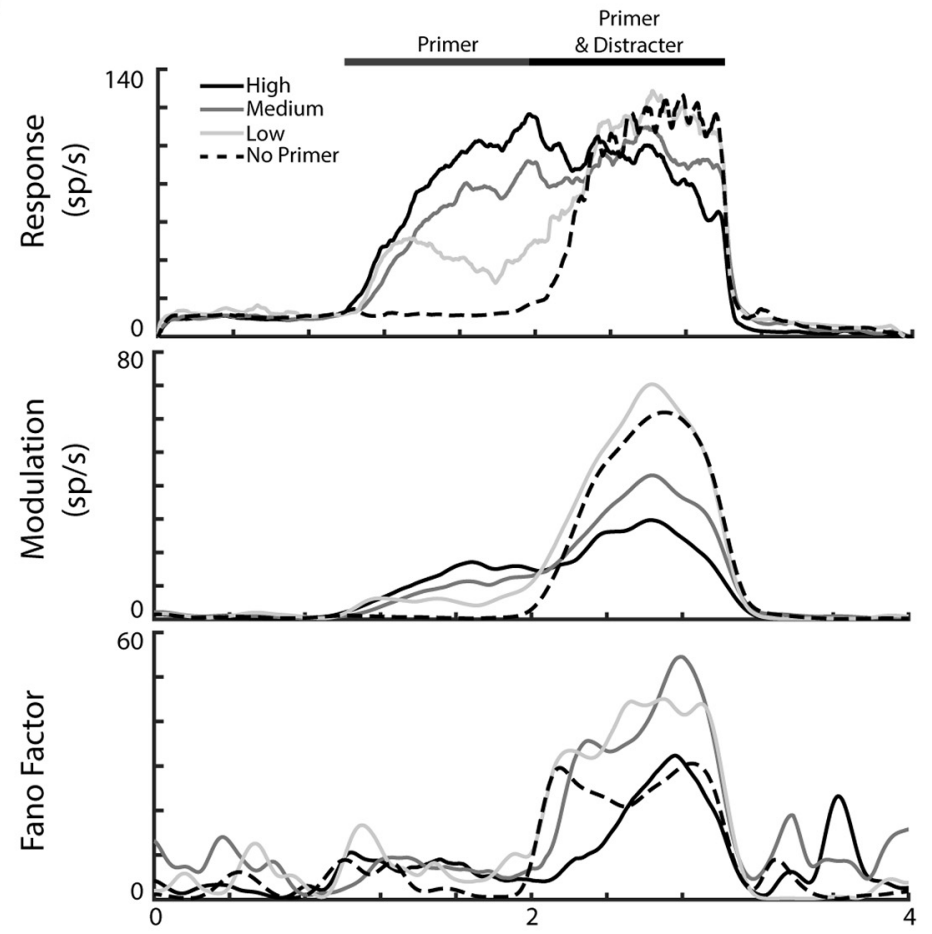

C

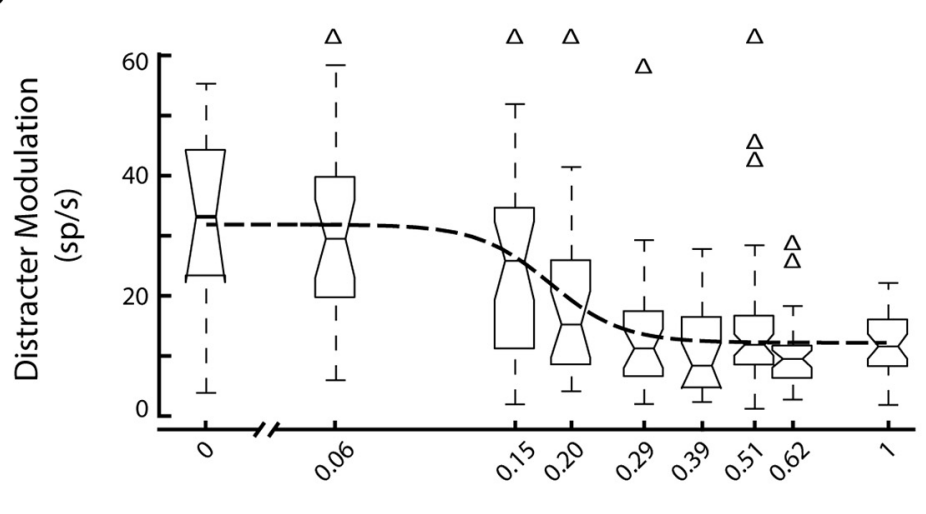

Primer Contrast (Weber)

Figure 6. Selective attention in CSTMD1 can lock on to a lower contrast target, ignoring the novel, high contrast distracter. $\boldsymbol{A}$, Stimulus pictograms and single-trial example traces from the same CSTMD1 for low-, medium-, and high-contrast primer conditions. Left, Responses when CSTMD1 locked-on to the primer and the presence of the distracter is ignored. Right, CSTMD1 responds to the distracter, once it is presented. $\boldsymbol{B}$, Top, Average inverse interspike interval across all trials ( $n=220$ across 7 dragonflies), separated by primer condition (none, low, medium and high) Middle, Average modulation across all trials, separated by condition. In the primer and distracter period, rank ordering of the conditions is inverted compared with $\boldsymbol{B}$. Top, Strong modulation in the ence of any frequency modulation during the paired presentation would indicate the distracter being selected. Primers were presented at constant low (0.06), medium (0.15) or high (0.51) Weber contrast, pseudo-randomly located at spatial locations $\mathrm{T}_{1}$ or $\mathrm{T}_{2}$ (Fig. $6 \mathrm{~A}$, primer at $\mathrm{T}_{2}$ location shown). The high contrast primer was set at 0.51 to be equiluminant with the average contrast (over time) of the frequency tagged distracter. Figure $6 \mathrm{~A}$ shows example responses of an individual CSTMD1 to these stimulus conditions, both when the lower contrast, primer trajectory retains selection and when selection switches to the high-contrast distracter. This shows that there can be trial-by-trial variability in which one of the targets was selected, either the continuing primer or the novel distracter.

Figure $6 B$ shows the average spike activity across all trials within a primer contrast condition (Fig. $6 B$, top). As expected, over the primer-alone period, the neuronal response increases with increased contrast (O'Carroll and Wiederman, 2014). When there is only the high contrast distracter (no-primer, dashed line) we observed the strong distracter response, subtly modulated by the frequency tagging technique. For the low and medium contrast primer conditions, responses trend toward the distracter response (as a proportion of trials). Interestingly, a high contrast primer sees a lower response over this duration, attributable to hyperpolarization observed from sustained firing.

We calculated the mean wavelet slice at the distracter frequency $\left(F_{\mathrm{d}}\right)$ across all trials for the same condition (Fig. $6 B$, middle). This reveals the magnitude of frequency-modulation induced by the distracter, at each point in time. In the primer alone period, modulation increases with primer contrast due to more power in the noise component. Over the primer \& distracter period the rank ordering of conditions is inverted compared with the primer only period in Figure $6 B$, top. This inversion indicates that the conditions with the most aggregate power at $\mathrm{F}_{\mathrm{d}}$ are the no-primer and low-contrast conditions, followed by the medium- and

\section{$\leftarrow$}

low-contrast and no-primer conditions (i.e., distracter selection) and weak modulation in the high-contrast condition (primer selection). Bottom, A stronger Fano factor reveals more variability of modulation, indicative of increased switching at lower primer contrasts. C, In an individual CSTMD1 recording, we assayed across a large range of primer contrasts, revealing a sigmoidal contrast sensitivity function $(n=217$ in 1 dragonfly). $\Delta$ indicate outliers. 
high-contrast conditions, respectively. This shows that the distracter is selected at a higher proportion of trials in no-primer and low-contrast primer conditions.

Due to the biasing effect of the primer (Fig. 5), we expect more distracter modulation in the no-primer (dashed line) and lowcontrast conditions, and the least when neuronal response locks-on to the high contrast primer, that is, no distracter modulation (black line). We observe this effect: increased primer contrast is associated with less response in the frequency domain, indicating fewer responses to the distracter target. Statistically, we observed a significant reduction in distracter modulation in the medium $(p=0.006)$ and high $(p<0.001)$ contrast group, but not the low-contrast group $(p=0.755)$, compared with the no primer group.

These data are averaged and therefore do not show the amount of intertrial variability associated with previously observed "rare" switching events between two equally salient targets (Fig. 4, see also Wiederman and O'Carroll, 2013). The Fano factor of the modulation is a measure of the trial by trial neuronal variability (Fig. 6B, bottom). The two conditions that exhibit the highest variability over the primer \& distracter period are the low and medium contrast conditions. This indicates that low and medium contrast conditions have the highest rates of betweentrial variability (i.e., on some trials the continuation of the primer is selected, while on other trials the distracter is selected) and within-trial variability (i.e., switching during the trial period). In comparison, the no primer and high contrast primer conditions have less variability as either the distracter or continuing primer is selected, respectively. The variability in the high contrast condition rises over time (Fig. 6B, bottom, solid black line), revealing an increase in the probability of switching targets over time. This is consistent with the finding that the effect of a primer diminishes over time (Fig. 5C).

In one long CSTMD1 recording, we were able to assay across a large range of primer contrasts (Fig. 6C). In this individual example, the sigmoidal function reveals that in a large proportion of trials, CSTMD1 locks-on to primer targets presented well below the average contrast of the introduced distracter $(0.51)$.

Both the aggregate data and the individual example reveal that CSTMD1 frequently locks-on to lower contrast targets (medium, 0.16 ), selecting them even in the presence of the high contrast distracter (mean 0.51). The mechanism underling this neuronal selective attention thus cannot be a "simple" winner-takes-all network unless evoking the competitive selection over sluggish temporal dynamics.

Intriguingly, responses to the low-contrast targets continuing along the primer trajectory are not associated with an increase in spike rate as would be expected by models of attention where low-contrast stimuli are attended by neuronally boosting the response to achieve competitive advantage against high-contrast distracters (Reynolds and Desimone, 2003). Instead, even when responding to low-contrast stimuli in the presence of a highcontrast distracter, CSTMD1 encodes the absolute strength of the attended target as if the distracter was not present (Fig. 6A). This could be critically important in behavior where a target is selected for pursuit amid a swarm, where absolute rather than relative activity might underlie the closed-loop control system.

\section{Modeling the neuronal processing underlying target responses}

What mechanism best explains the measured data? To test this, we developed six algorithmic models. The six models included two models that assumed shared attention (including one with
A
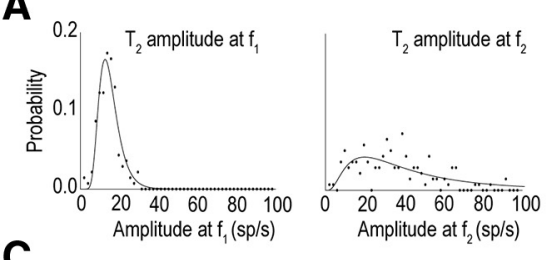

B
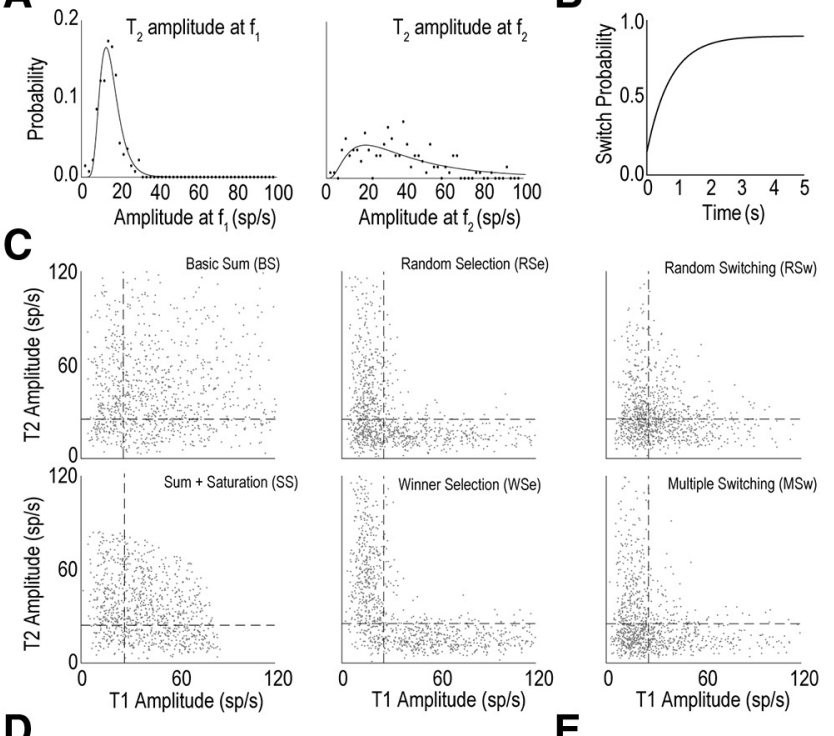

E
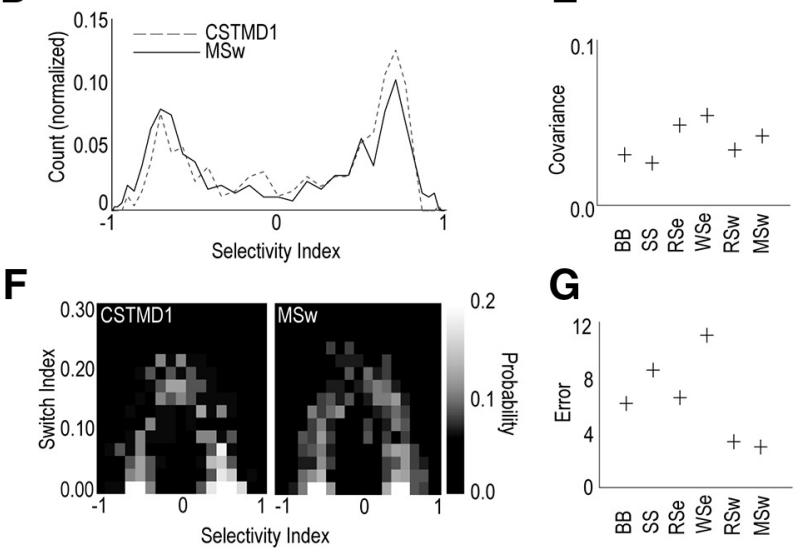

G

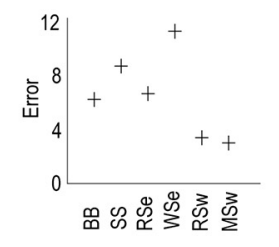

Figure 7. A switching model matches the physiological data. A, Power distributions for frequency responses from $\mathrm{T}_{2}$ at $\mathrm{f}_{1}$ (left) and $\mathrm{f}_{2}$ (right) calculated from recorded trials. Modeled trial data were randomly selected from these power distributions representing the power contribution of each target. $\boldsymbol{B}$, Switch probability as time progresses for multiple switching (MSW). Initially the likelihood of switching is low before rising to $90 \%$. After a switch, the switch probability resets permitting more to occur. C, Example scatter plots for each of the six models tested: summation (top left), summation with saturation (bottom left), random selection (top middle), higher power always wins selection (bottom middle), random switching (top right) and multiple switch model (bottom right). These scatterplots can be compared with the phys iological data in Figure 2B. D, Histogram of selectivity index for recorded CSTMD1 data and model output. Real data curves are cross correlated with model-derived curves to generate covariance score. $\boldsymbol{E}$, Covariance of the six models against CSTMD1 data from the histogram analysis. Higher covariance (i.e., model WSe) is indicative of a more representative model. $\boldsymbol{F}$, Two-dimensional histogram that accounts for selectivity and switching for CSTMD1 (left) and MSw model (right). G, Error calculated as RMS deviations (2D histograms) from the six models, each against recorded CSTMD1. Low values indicate the most representative model of both CSTMD1 selection (Fig. 2B) and switching (Fig. 4B).

saturation), two models that applied selection and two models which applied selection with switching. For input to these models we collected the response modulation amplitude from the wavelet analysis for the single target trials (i.e., $\mathrm{T}_{1}$-only or $\mathrm{T}_{2}$-only) (Fig. 2). From this we produced four lists $\left(T_{1} f_{1}, T_{1} f_{2}, T_{2} f_{1}, T_{2} f_{2}\right)$ representing the response modulation amplitude at the target's flicker frequency and at the comparison frequency (i.e., no modulation). We binned these responses and fit a log-normal distribution to each target and frequency pair (T2 examples are shown in Fig. 7A). We then infinitely sampled from these model distri- 
butions to generate an arbitrary number of synthetic target responses.

To simulate switching, we generated a $1 \mathrm{~s}$ time course of response modulation for testing all models, equivalent to taking a 1D slice from the CWT analysis (as in Fig. 3). For realism, we added Gaussian white noise ( 5 spikes/s max width) and smoothed the data using a $0.2 \mathrm{~s}$ average filter. For switching models, this smoothing was done after calculating the switch. This produced waveforms qualitatively similar to those observed from taking a single-frequency slice of a CWT, including switch transitions (Fig. 3C).

We sampled from the distribution 1000 times for each pairing $\left(T_{1} f_{1}, T_{1} f_{2}, T_{2} f_{1}, T_{2} f_{2}\right)$. Each model used a combination of these to generate output responses for both $f_{1}$ and $f_{2}$. Basic summation (BS) assumed that the output power at both $\mathrm{f}_{1}$ and $\mathrm{f}_{2}$ were the corresponding powers of the input target (i.e., $T_{1} f_{1}$ and $T_{2} f_{2}$ ). Saturating summation (SS) summed like BS, then applied a soft saturation to reduce the overall modulation power evenly between $\mathrm{f}_{1}$ and $\mathrm{f}_{2}$ (maximum power of 100 spikes/s). Random selection (RSe) randomly selected either $\mathrm{T}_{1}$ or $\mathrm{T}_{2}$ and used that target's corresponding power for $\mathrm{f}_{1}$ and $\mathrm{f}_{2}$ (i.e., if $\mathrm{T}_{1}$ was selected the frequency responses would be $\mathrm{T}_{1} \mathrm{f}_{1}$ and $\left.\mathrm{T}_{1} \mathrm{f}_{2}\right)$. Winner selection (WSe) selected a "winner" target with the greatest modulated power, with the assumption that modulation was proportional to the target response (if $\mathrm{T}_{1} \mathrm{f}_{1}>\mathrm{T}_{2} \mathrm{f}_{2}, \mathrm{~T}_{1}$ would be selected and vice versa). Random switching ( $\mathrm{RSw}$ ), randomly selected an initial target (as per RSe) however assumed that a switch occurred in a percentage of trials at some point during the trial's duration. Multiple switching (MSw) assumed a more sophisticated switching rate, allowing the system to switch multiple times. The switch probability was defined by the following formula:

$$
P(\text { switch })=S-\tau e^{-t / \tau}
$$

Where $S$ represents the probability that a switch never occurs and $\tau$ represents the rate of increase of switching over time (Fig. $7 B$ ).

The outputs of all six models are shown in Figure 7C. The summation model (BS) populates all four quadrants (including in the "shared or switch" zone of Fig. 2B). This combination of taking power from both targets together does not match the electrophysiological results (Fig. 2B). Both selection models (RSe and WSe) adhere far closer to the distribution seen in Figure 2 except that the shared zone is too sparsely populated. The switching models qualitatively match the physiological data with a bias to $\mathrm{T}_{1} / \mathrm{T}_{2}$ only responses (the $\mathrm{L}$ shape) but with proportion of shared zone responses indicative of switching.

To assess each model quantitatively, we generated the frequency polygon (Figs. 2, 4) of the selectivity index values calculated from the model outputs. An example of the response of the MSw model (gray line) compared with the electrophysiological data (dotted line) is shown in Figure $7 D$. With cross-correlation, we compared each model's frequency polygon with CSTMD1's (derived from Fig. 2C).

Via this metric, both selection models (RSe, WSe) provided the best match to the recorded data (Fig. 7E). However, this selection metric ignores the switching behavior inherent in the model. To test whether pure selection was sufficient to explain the data, we used the model outputs to calculate the "switch index" (Fig. 4) for each model's responses. We binned these data to generate a $2 \mathrm{D}$ histogram (Fig. $7 F$ ). We repeated this process for the electrophysiological data and calculated the RMS error between them. As both switching models had free parameters (i.e., probability of switching) we optimized both these models against this RMS error. The RSw model was most successful with a $100 \%$ probability of a switch at a random time during the trial. The MSw model was optimal with a $90 \%$ switch probability and $0.75 \mathrm{~s}$ time constant. When accounting for both CSTMD1's selection and switching, the (MSw) model had the least error (Fig. $7 G$ ).

Summation models (BS, SS) generated too many responses in the shared zone by increasing overall power, while Selection models (RSe, WSe) eliminated shared zone responses entirely. Switching models (RSw, MSw) provided the appropriate compromise, encapsulating the selection responses in top-left and lower-right quadrants and generating some shared zone responses due to switching. Therefore, the parsimonious explanation for our observations is modeled with a process that selects a single target but is capable of switching one or more times during a trial.

\section{Discussion}

Our novel approach of analyzing frequency tagged, intracellular spike trains allowed us to verify the presence of selective attention in CSTMD1 (Wiederman and O'Carroll, 2013), and build on this result by reliably identifying which target of a pair was selected at any moment in time. Additionally, as frequency tagging does not rely on an inhomogeneous receptive field to differentiate targets, this technique affords more freedom in experimental design and potential application to STMD neurons with either smaller or more homogeneous receptive fields. We leveraged this to design a set of experiments that probe the properties of selective attention in the context of low-contrast priming and abrupt-onset distracter presentation, thus moving beyond the capabilities of the technique presented in Wiederman and O'Carroll (2013).

Despite these advantages, on $\sim 25 \%$ of trials regardless of stimulus conditions, levels of frequency modulation were belownoise, even with the stimulus generating spiking responses. Flickering targets located within the strongest parts of the receptive field may reach saturation during the low-contrast phase of the stimulus, resulting in a lack of modulation (i.e., clipping). Conversely, frequency tagged targets presented in less sensitive regions of the receptive field may not elicit strong enough modulation over the carrier signal. Both CSTMD1's saturation and sensitivity may vary over time and between animals. In future experiments, these effects might be minimized by dynamically changing the stimulus waveform, decreased or increased to account for saturation or sensitivity respectively.

Although frequency-tagging was used as an identifier, could the frequency itself interact with facilitatory or selective processing? Such a factor can play a role in other animal models, with honeybees preferencing 20-25 Hz visual flicker and avoiding 2-4 $\mathrm{Hz}$ (Van De Poll et al., 2015). Even a single luminance change is enough to break inattentional blindness in humans (Palmer et al., 2018). To minimize this possibility, we distributed the two tagging frequencies across two spatial locations $\left(\mathrm{T}_{1}\right.$ and $\left.\mathrm{T}_{2}\right)$ as well as testing our entire experimental paradigm at two different frequency tagged pairs. Throughout these experiments, we did not observe any effect of the frequency tagging beyond our intended purpose as an identification technique.

Attention is a limited resource (Alvarez and Franconeri, 2007), therefore animals across species are motivated to guide the deployment of attention in an ethologically meaningful and efficient way. One guide is spatial or temporal cueing, often through inhibitory neural mechanisms (Römer et al., 2002; Ruthruff and Gaspelin, 2018). For example, Drosophila are more likely to orient toward cued locations of the receptive field when subsequently presented with multiple targets (Sareen et al., 2011). 
Female crickets prefer leading male auditory signals to signals arriving later (Snedden and Greenfield, 1998; Römer et al., 2002), suggesting an inherent bias toward 'locking on' to the first stimulus and ignoring those subsequent. This is similar to what we have observed in CSTMD1, with the priming by a preceding target biasing selection to those that continue along the projected trajectory. This property we termed 'predictive gain modulation' and our recent experiments reveal that even short duration primers can elicit robust predictive gain (Fabian et al., 2019). The interactions between mechanisms underlying prediction and those of selective attention are a focus of our future research.

In CSTMD1, the effect of spatiotemporal cueing was so strong that even targets of lower visual salience can win over an abruptonset, high-contrast distracter. In attentional networks, saliency is a prominent attribute for guiding selection and seems to innately capture attention. This leads to a conundrum; if the most salient targets were to capture attention moment-to-moment, then the system might too often be distracted from any given task. For example, will the dragonfly ever feed if the prey of constantly varying contrast (i.e., moving against a cluttered background) are dynamically more or less salient than others in the swarm? Conversely, the onset of a novel salient stimulus may signal the necessity to attend to a new event or abandon the current task completely in favor of survival behavior (e.g., an approaching bird).

Our results bear resemblance to behavioral results in Drosophila (Koenig et al., 2016). Tethered flies in an arena were presented with a pair of vertical lines equally offset from the flies' midline. Flies made a decision to respond to either one line or the other by turning to bring it into the midline. In subsequent trials, these flies displayed a bias for turning toward the originally selected stimulus and ignoring the alternative. However, over time this bias was lost. The mean 'attention span' (time before the bias was lost) was $4 \mathrm{~s}$ in wild-type flies, but reduced to $1 \mathrm{~s}$ in mutants defective in selective attention. Active switching between competing stimuli may be indicative of endogenous drive by topdown control mechanisms (Miller et al., 2012). van Swinderen (2012) found that, in Drosophila, a minimum amount of time must pass between the original selection of a target and switching to a new stimulus, and switching at all was reliant on short-term memory genes.

In human psychophysics, both abrupt-onset (Yantis and Jonides, 1984) and perceptually new objects (Franconeri et al., 2005) provoke attentional capture, a phenomenon where attention is automatically and involuntarily directed at a particular, often task irrelevant, feature (Remington et al., 1992). In our CSTMD1 recordings, we found no evidence for attentional capture. Instead, the earliest period of the paired-targets revealed the strongest bias to the previous primer trajectory, with the possibility of switching to the more novel distracter at a later time. Thus, rather than attending to a novel distracter, this system locked-on to the expected target trajectory. CSTMD1 predicts future target location, even following an occlusion, with an enhancement in front of the prior path and suppression in the surround (Wiederman et al., 2017; Fabian et al., 2019). During the initial window, the continuing target is facilitated (gain increase) by the preceding target and continuously moving into its self-generated spotlight of predictive gain. However, the distracter appears within the suppressed surround (gain decrease) and therefore will not elicit attentional capture, similar to some recent findings in Psychophysics (Ruthruff and Gaspelin, 2018). By the middle period, the distracter may itself have self- facilitated, enabling a more even competition for target selection and thus increasing the probability of a switch. Whether this self-facilitation occurs at both target locations before selection, or only at the single selected location is currently under investigation.

The possibility that non-selected stimuli also generate a spotlight of neuronal gain modulation is similar to proposed mechanisms underlying attention in primates (Reynolds and Desimone, 2003). Primate cortical cells are thought to be 'hardwired' to respond to the highest contrast stimulus, a property that can be exploited by attentional systems (Schiller and Lee, 1991; DeWeerd et al., 1999). Here the representation of stimuli is modulated by enhancing the effective contrast of the focus of attention (Martinez-Trujillo and Treue, 2002; Reynolds and Desimone, 2003). Through this enhancement, less salient and even nonpreferred stimuli can come to dominate the response of neurons in V4 (Reynolds and Desimone, 2003), MT, and MST (Recanzone et al., 1997; Treue and Maunsell, 1999). However, it is important to note that, unlike in primates, CSTMD1 encodes the absolute strength of the selected stimulus-this includes encoding a low-contrast target with a low firing rate, even as that stimulus is selected despite the presence of a high-contrast distracter simultaneously presented within the receptive field (Fig. $6 A$, middle left).

This neuronal enhancement observed in primates may be mechanistically similar to the predictive gain modulation observed in CSTMD1, where in response to a single target gain is increased ahead of the prior path and suppressed in the surround. In primates it is the presence of distracters that triggers this attentional enhancement (Treue and Maunsell, 1999; Reynolds et al., 2000; Treue, 2001; Reynolds and Dismone, 2003). However, in CSTMD1, facilitation (i.e., the local gain increase) enhances the neuronal response to even an individually presented single target (Dunbier et al., 2012; Wiederman et al., 2017). In the presence of distracters the facilitated strength of the selected target is retained as if the distracter did not exist.

How might the dragonfly brain use information represented by CSTMD1? In order for CSTMD1 to be behaviorally relevant for tracking targets through space, the spatial location of the selected target must be recovered from either CSTMD1, or a population of similarly tuned neurons. It should be noted that CSTMD1 is not necessarily the only neuron that inherits properties of both prediction and selection, presumably formulated in presynaptic networks. It is possible that the precise location of a target represented by an array of neurons with ambiguous responses, though with overlapping receptive fields, is calculated with divisive normalization (Evans et al., 2016). Although this has not been demonstrated in the dragonfly, normalization is a common neuronal computation that has been observed in a variety of brain systems and taxa (Carandini and Heeger, 2012).

The facilitation effect observed in CSTMD1 spreads ahead of a target along a straight trajectory (Wiederman et al., 2017), thus predicting that a tracked target will continue moving in a straight line relative to the dragonfly. This prediction matches ethological goals, where the dragonflies' main method of pursuit is an interception path (Mischiati et al., 2015) from behind and below the target (Olberg et al., 2007) perhaps even using motion camouflage, where the target is kept in a stable position relative to the pursuing dragonfly (Mizutani et al., 2003). During predatory pursuit dragonflies fixate targets in a high-resolution optical fovea situated on the dorsal surface of the eye (Olberg et al., 2007; Mischiati et al., 2015). CSTMD1 responses are greatest for targets that move upward and toward the periphery (away from the midline) thus may be involved in error signals driving move- 
ments that preserve the retinal position of the selected target in the optical fovea. Functional roles for CSTMD1 (and other STMD neurons) in such closed-loop pursuit scenarios still remain speculative, however we model these target-detection pathways in virtual-reality, computational simulations to elucidate these complex interactions (Bagheri et al., 2017a,b).

The ability of a neuron to respond with the same strength to a target presented alone, or when selected in a pair, is likely to underlie the dragonfly's exceptional ability to hunt in swarms (Combes et al., 2012). Such neuronal processing may have evolved to overcome the confusion effect by singling-out targeted prey amid a swarm (Landeau and Terborgh, 1986). Behavioral studies in some dragonfly species, e.g., Libellula adults (Combes et al., 2012) and nymphs (Jeschke and Tollrian, 2007), show that they are adept at hunting in swarms throughout life. Although not tested in Hemicordulia, this hawking dragonfly would also likely benefit from neuronal processing that reduces the confusion effect via selective attention, as they spend most of their adult life hunting and patrolling territory on the wing and can regularly be observed hunting amid swarms of prey and conspecifics.

\section{References}

Alvarez GA, Franconeri SL (2007) How many objects can you track?: Evidence for a resource-limited attentive tracking mechanism. J Vis 7:14.110.

Asadollahi A, Mysore SP, Knudsen EI (2010) Stimulus-driven competition in a cholinergic midbrain nucleus. Nat Neurosci 13:889-895.

Bagheri ZM, Cazzolato BS, Grainger S, O'Carroll DC, Wiederman SD (2017a) An autonomous robot inspired by insect neurophysiology pursues moving features in natural environments. Journal of Neural Engineering 14:046030.

Bagheri ZM, Wiederman SD, Cazzolato BS, Grainger S, O'Carroll DC (2017b) Performance of an insect-inspired target tracker in natural conditions. Bioinspiration and Biomimetics 12:025006.

Baird JM, May ML (1997) Foraging behaviour of Pachydiplax longipennis (Odonata: Libellulidae). Journal of Insect Behavior 10:655-678.

Carandini M, Heeger DJ (2012) Normalization as a canonical neural computation. Nat Rev Neurosci 13:51-62.

Combes SA, Rundle DE, Iwasaki JM, Crall JD (2012) linking biomechanics and ecology through predator-prey interactions: flight performance of dragonflies and their prey. J Exp Biol 215:903-913.

de Bivort BL, van Swinderen B (2016) Evidence for selective attention in the insect brain. Current Opinion in Insect Science 15:9-15.

DeWeerd P, Peralta MR 3rd, Desimone R, Ungerleider LG (1999) Loss of attentional stimulus selection after extrastriate cortical lesions in macaques. Nat Neurosci 2:753-758.

Dunbier JR, Wiederman SD, Shoemaker PA, O'Carroll DC (2012) Facilitation of dragonfly target-detecting neurons by slow moving features on continuous paths. Front Neural Circuits 6:79.

Edman JD, Haeger JS (1974) Dragonflies attracted to and selectively feeding on concentrations of mosquitoes. Fla Entomol 57:408.

Evans BJE, O'Carroll DC, Fabian JM and Wiederman SD (2019) Differential tuning to visual motion allows robust encoding of optic flow in the dragonfly. J Neurosci. Advance online publication. Retrieved Sep 3, 2019. doi:10.1523/JNEUROSCI.0143-19.2019.

Evans BJE, O'Carroll DC and Wiederman SD (2016) Salience invariance with divisive normalization in higher-order insect neurons. In $20166^{\text {th }}$ European Workshop on Visual Information Processing, pp 1-6. Marseille: IEEE.

Fabian JM, Dunbier JR, O'Carroll DC, Wiederman SD (2019) Properties of predictive gain modulation in a dragonfly visual neuron. J Exp Biol 222: jeb207316.

Feldman JA, Ballard DH (1982) Connectionist models and their properties. Cognitive Science 6:205-254.

Franconeri SL, Hollingworth A, Simons DJ (2005) Do new objects capture attention? Psychol Sci 16:275-281.

Geurten BR, Nordström K, Sprayberry JDH, Bolzon DM, O'Carroll DC (2007) Neural mechanisms underlying target detection in a dragonfly centrifugal neuron. J Exp Biol 210:3277-3284.
Ghose GM, Maunsell JH (2008) Spatial summation can explain the attentional modulation of neuronal responses to multiple stimuli in area V4. J Neurosci 28:5115-5126.

Harrison IT, Weiner KF, Ghose GM (2013) Inattention blindness to motion in middle temporal area. J Neurosci 33:8396-8410.

Hausen K (1982) Motion sensitive interneurons in the optomotor system of the fly. II. The Horizontal Cells: Receptive field organization and response characteristics. Biol Cybern 46:67-79.

Jeschke JM, Tollrian R (2007) Prey swarming: which predators become confused and why? Animal Behaviour 74:387-393.

Koenig S, Wolf R, Heisenberg M (2016) Vision in flies: measuring the attention span. PLoS One 11:e0148208.

Labhart T, Nilsson DE (1995) The dorsal eye of the dragonfly sympetrum: specializations for prey detection against the blue sky. J Comp Physiol [A] 176:437-453.

Landeau L, Terborgh J (1986) Oddity and the 'confusion effect' in predation. Animal Behaviour 34:1372-1380.

Martinez-Trujillo JC, Treue S (2002) Attentional modulation strength in cortical area MT depends on stimulus contrast. Neuron 35:365-370.

Miller SM, Ngo TT, van Swinderen B (2012) Attentional switching in humans and flies: rivalry in large and miniature brains. Frontiers in Human Neuroscience 5:188

Mischiati M, Lin HT, Herold P, Imler E, Olberg R, Leonardo A (2015) Internal models direct dragonfly interception steering. Nature 517:333-338.

Mizutani A, Chahl J, Srinivasan MV (2003) Motion camouflage in dragonflies. Nature 423:604.

Nityananda V (2016) Attention-like processes in insects. Proc Biol Sci 283: 20161986

Norcia AM, Appelbaum LG, Ales JM, Cottereau BR and Rossion B (2015) The steady-state visual evoked potential in vision research: a review. J Vis 15:4.

Nordström K, Bolzon D, O'Carroll DC (2011) Spatial facilitation by a highperformance dragonfly target detecting neuron. Biology Letters 7: 588 592.

O'Carroll DC (1993) Feature-detecting neurons in dragonflies. Nature 362: 541.

O'Carroll DC, Wiederman SD (2014) Contrast sensitivity and the detection of moving patterns and features. Philos Trans R Soc Lond B Biol Sci 369:20130043.

Olberg RM, Worthington AH, Fox JL, Bessette CE, Loosemore MP (2005) Prey size selection and distance estimation in foraging adult dragonflies. J Comp Physiol [A] 191:791-797.

Olberg RM, Seaman RC, Coats MI, Henry AF (2007) Eye movements and target fixation during dragonfly prey-interception flights. J Comp Physiol [A] 193:685-693.

Palmer DB, Yamani Y, Bobrow TL, Karpinsky ND, Krusienski DJ (2018) Transient signals and inattentional blindness in a multi-object tracking task. i-Perception 9:2041669518754595.

Pollack GS (1988) Selective attention in an insect auditory neuron. J Neurosci 8:2635-2639.

Recanzone GH, Wurtz RH, Schwarz U (1997) Responses of MT and MST neurons to one and two moving objects in the receptive field. J Neurophysiol 78:2904-2915.

Remington RW, Johnston JC, Yantis S (1992) Involuntary attentional capture by abrupt onsets. Percept Psychophys 51:279-290.

Reynolds JH, Desimone R (2003) Interacting roles of attention and visual salience in V4. Neuron 37:853-863.

Reynolds JH, Pasternak T, Desimone R (2000) Attention increases sensitivity of V4 neurons. Neuron 26:703-714

Römer H, Hedwig B, Ott SR (2002) Contralateral inhibition as a sensory bias: the neural basis for a female preference in a synchronously calling bushcricket, Mecopoda elongata. Eur J Neurosci 15:1655-1662.

Ruthruff E, Gaspelin N (2018) Immunity to attentional capture at ignored locations. Atten Percept Psychophys 80:325-336.

Sareen P, Wolf R, Heisenberg M,, (2011) Attracting the attention of a fly. Proc Natl Acad Sci U S A 108:7230-7235.

Schiller PH, Lee K (1991) The role of the primate extrastriate area V4 in vision. Science 251:1251-1253.

Simons DJ, Chabris CF (1999) Gorillas in our midst: sustained inattentional blindness for dynamic events. Perception 28:1059-1074.

Snedden WA, Greenfield MD (1998) Females prefer leading males: relative 
call timing and sexual selection in katydid choruses. Anim Behav 56:1091-1098.

Tang S, Juusola M (2010) Intrinsic activity in the fly brain gates visual information during behavioural choices. PLoS One 5:e14455.

Treue S (2001) Neural correlates of attention in primate visual cortex. Trends Neurosci 24:295-300.

Treue S, Maunsell JHR (1999) Effects of attention on the processing of motion in macaque middle temporal and medial superior temporal visual cortical areas. J Neurosci 19:7591-7602.

Van De Poll MN, Zajaczkowski EL, Taylor GJ, Srinivasan MV, van Swinderen B (2015) Using an abstract geometry in virtual reality to explore choice behaviour: visual flicker preferences in honeybees. J Exp Biol 218: $3448-3460$ van Swinderen B (2012) Competing visual flicker reveals attention-like rivalry in the fly brain. Front Integr Neurosci 6:96.

Wiederman SD, O'Carroll DC (2013) Selective attention in an insect visual neuron. Curr Biol 23:156-161.

Wiederman SD, Fabian JM, Dunbier JR and O'Carroll DC (2017) A predictive focus of gain modulation encodes target trajectories in insect vision. eLife 6:e26478.

Wiederman SD, Shoemaker PA, O'Carroll DC (2013) Correlation between OFF and ON channels underlies dark target selectivity in an insect visual system. J Neurosci 33:13225-13232.

Yantis S, Jonides J (1984) Abrupt visual onsets and selective attention: evidence from visual search. J Exp Psychol Hum Percept Perform 10:601621. 